not obstruct vision. The electrode is larger, obstructs vision more and is not so easily handled. The acid in most cases is painless and can be applied without the patient knowing it.

I shall mention a few only of the drugs used in such cases. Iodoform and boric acid I have already mentioned as suitable dressings, but they have no influence over the growth of polypi. Tannic acid, alum, etc., are worse than useless. Argentic nitrate may be used to stop a discharge of pus, but I am sure that it stimulates the growth of polypi and of granulations. Hydrogen peroxid is useful only as a cleansing agent and is inferior to a solution of bichlorid of mercury. Notwithstanding all that has been said of alcohol, I believe that it has absolutely no influence over the growth of polypi or granulations, but it does lessen the discharge of pus. Injection of the growth with Monsel's solution is effective, if well done, but is painful and can be used on large poly pi only. Patients will ask us if the growth will return and we ask ourselves what shall be done to prevent a recurrence.

Now, I think we are all of the opinion that suppurative disease or caries is always the cause of polypi and of granulations. It is therefore necessary to stop the suppuration and remove all necrotic tissue before we can say that our patient is cured, for polypi and polypoid proliferations will not grow in dry soil like sage brush and cacti in our western deserts, but like toadstools, they require shade and moisture, and this moisture is pus.

Let us always put in practice that old, sound surgical principle that wherever there is pus, let it out if possible and establish free drainage. If the growth returns and if the perforation is small, we should not hesitate to make it larger and when necessary remove one or mole of the ossicles, thus establishing free drainage and giving us an opportunity to treat the diseased parts effectually ; always remembering, however, that it is not a trifling operation, but must be skilfully performed and subsequently carefully treated, for cases of meningitis following it are not unknown and $I$ think we are too apt to cover our failures and publish our successes.

\section{TUMOR OF THE BRAIN INVOLVING THE OCULAR NERVES.}

Specimen presented to the Medical Society of Washington, D. C. BY ClaARNCE R. DUfOUR, Phar. D., M.D. WASHINGTON, D. C.

Physician in Charge of Eye Department Eastern Dispensary; Ophthalmologist and Otologist to Sibley Memorial Hospital; Assistant in Eye pital, and Instructor in Oph thalmology and Otology, Georgetown Medical College.

Mrs. H., widow, age about 56 years was referred to me at the Woman's Clinic, in the summer of 1894, on account of her eyes. Upon examination I found a complete paralysis of the muscles supplied by the third, fourth and sixth nerves, an exophthalmus and optic atrophy, all on left side. I diagnosed the trouble as being in the brain and so told the daughter who accompanied her. I could obtain no specific history. She had been operated upon some months previous for empyema of left antrum, the opening being made in the cavity of second molar tooth; there was free drainage through the nostril when I saw her. I kept the antrum well washed out with sol. boric acid and gave her sat. sol. iodid of potas. sium, $10 \mathrm{gtt}$. three times daily, increasing one drop daily, until she was taking $30 \mathrm{gtt}$. three times daily. Any attempt to increase this amount produced such constitutional disturbance that it could not be done. She was kept on this treatment until complete symp. toms of iodism were manifested, when it was changed to $1.16 \mathrm{gr}$. bichlorid of mercury and $3 \mathrm{grs}$. potassium iodid three times daily, and continued for months with occasional intermiseion of a few days. No result whatever from the treatment. About the middle of the summer she began to complain of intense pain in her head, which at first yielded to anodype treatment but which soon became constant and nothing but morphia would allay. About this time there began to be symptoms of loss of sensation on side of face and anesthesia of cornea, indicating that the first division of the fifth nerve was being implicated. This condition continued, with no abatement of the symptoms; she had periods of hallucinations, and as her daughter was obliged to work away from home, and there being no one to leave the mother with, the latter was sent to the hospital. It was thought best to reopen the antrum so as to establish freer drainage; this was done, considerable pus escaping. It was then washed out with antiseptic solution two to three times daily. An examination of the urine was made with results as follows: Amber in color, cloudy ppt., acid reaction; small amount of aibumin, epithelial cells and pus were found. After operation on antrum the pain in head subsided for a few days but began again and continued with occasional periods of intermission. Sine was discharged from hospital in the early part of November. Her condition grad. ually became worse, her right eye began to show symptoms of incipient atrophy of the optic nerve and an external squint was manifested. She was able to go about until April 1895 when she had to go to bed. She grew worse, her mind wandered and word deafness became manifest. On May 3 she died. On the following morning the skull was opened and the brain removed; It was put into a 2 per cent. solution of formalin, and a few days later the examination revealed the following condition: Gumma of dura mater in the anterior part of left middle fossa; this involved by extension the left anterior temporo.sphenoidal lobe; the growth surrounded the internal carotid artery, exerting pressure on the cavernous sinus and involved the left optic nerve at the commissural origin; considerable edema of the left anterior sphenoidal lobe. A second and smaller gumma involved that part of the brain mass which constitutes the left olfactory convolution; the third and smallest lay in the angle at the right optic commissure. The bone around the first growth had lost its compact covering and had become porous; the dura was very adherent to the bone. The ocular conditions during life were cleared up, I think, satisfactorily by the findinge at the post-mortem. The cavernous sinus receives anteriorly the ophthalmic vein through the sphenoidal fissure, and on its inner walls is found the internal carotid artery and the sixth nerve; on its outer wall are the third, fourth and the first division of the fifth nerve.

The pressure exerted by the tumor upon the sinus explains the paralysis of the muscles and the anesthesia of cornea, etc., this pressure together with pressure upon the ophthalmic vein, accounts for the exophthalmus by preventing the return flow of blood through the angular and ophthalmic veins into the cavernous sinus. The pressure also being exerted 
upon the optic nerve in front of the chiasm, was the cause of the optic atrophy of left eye. The edema of the temporo-sphenoidal lobe, would, I think, satisfactorily explain the word deafness. The atrophy of the right eye was due to the smallest tumor in the angle of the right optic commissure. The cause of the external strabismus is somewhat obscure, as there was no implication of the third nerve on that side; my opinion is that there was pressure exerted upon this nerve in the region of the sphenoidal fissure, probably as it passed through it. The case was referred to me by Dr. Heiberger, and the post-mortem was made by Dr. D. S. Lamb of the Army Medical Museum, of this city.

1016 I Street, N.W.

\section{OBSERVATIONS ON CONGENITAL DISLOCA-} TION OF THE HIP.

BY BERNARD E. BRODHURST, F.R.C.S.

SENIOR SURGEON TO THE ROYAL ORTHOPEDIC HOSPITAL, ETC. LONDON, ENG.

Congenital dislocations of the hip are for the most part traumatic. Displacements occur also, however, through deficient development.

Any obstruction at birth, whether through malformation of the pelvis or caused by the position of the child-the breech for instance, presenting, or the child or its head being unusually large, may occasion this dislocation.

In a breech presentation the thighs are flexed upon the abdomen, so that the head of the femur presses on the posterior and inferior portion of the capsule of the joint. But in this position, the application of the slightest force in downward traction will displace the head of the bone and cause it to pass away from the acetabulum and to lie upon its brim. And lying on the border of the cotyloid cavity, extension of the limb at birth is alone sufficient to displace the head beyond the brim. The blunt hook or the finger in the bend of the thigh, to facilitate and hasten the birth, may easily thus displace the head of the bone. And, indeed, such is the result of downward traction. A sensation of sudden yielding of the limb is felt by the hand of the accoucheur, as the head of the bone is displaced from its socket, and at the same time a click is heard. At that moment the caput femoris escapes from the acetabulum, and is found lying immediately beyond the brim of the cotyloid cavity. It is only after the weight of the child has been borne on the feet, as in standing and in walking, that the head of the femur arrives at the external iliac fossa. Shortening of the limb necessarily increases as the head of the femur becomes farther removed from the acetabulum and lies upon the dorsum of the ilium, and, equally, lameness increases.

Dr. Tyler Smith informed me that such was his experience in regard to these accidents at birth.

After my essay ${ }^{1}$ on this subject was published, I saw two cases of congenital dislocation with Dr. Tyler Smith. One was a boy, aged fifteen months, with dislocation of the left femur, and the other was a girl, aged five months, also with dislocation of the left femur. The boy was a well-developed and large child and the girl had a large head. There was not any other abnormality in either of these children. In both birth was considerably delayed; in the first by

\footnotetext{
1 St. George's Hospital Reports, vol. i, 1866.
}

a breech presentation, and in the second by the large head. In each case reduction of the dislocation was easily effected, and there was not any subsequent displacement.

Some imagine that these dislocations are not truly congenital because they are not observed at birth, nor until efforts are made to walk. Then, if the child falls as probably he will, it is supposed that in falling he has dislocated his hip. But dislocation does not occur in this manner. And without intending to mislead, parents are apt to connect the lameness with the fall, forgetting that the child has alwoys been more or less lame and unable to walk.

It has already been stated that children in whom this dislocation occurs are not infrequently unusually large. This was especially remarked with regard to two boys in St. George's Hospital. I admitted these boys as in-patients, each having congenital dislocation of both hips. One aged twelve years, was as big as a large boy of fourteen, and the other, aged fourteen was like a strong boy of sixteen or seventeen. So much was this the case that doubt was expressed on this question of age, and special inquiry was made, when the facts were verified. And in the case referred to by Mr. Holmes, ${ }^{2}$ and recorded at greater length by myself ${ }^{3}$ in which the right femur was dislocated, the child's head was of unusual size. Also in a case where I operated with Dr. De la Cour, and where both hips were dislocated the head was enormous.

But traumatic congenital dislocation does not alone occur at birth; it may also be occasioned in utero. $I^{4}$ have recorded several instances where, as the result of accident and shock, spasmodic action was set up, and this was followed by dislocation and fracture and distortion.

Cruveilhier ${ }^{5}$ has given a representation of a fullgrown fetus with luxation of the heads of the thighbones, and with clubbed hands and feet; and he observed that the heads of the thigh-bones, as well as the cotyloid cavities, were progressing to their normal development. Also, there is found in the hydrocephalic and the anencephalic fetus every form of luxation and subluxation, together with muscular retraction in every variety.

Grawitz ${ }^{6}$ records that in every case examined by him there was not only dislocation of the hip, but also club foot, club hands, scoliosis, ectopium abdominis, spina bifida, and other deformities and malformations present. And such is also the case with Mr. Lockwood's ${ }^{7}$ specimens; they are malformations and deformities due to arrest of development.

In pathologic museums dislocations, occurring whether in utero or at birth, whether alone or com. plicated with fracture or distortion, with malformation or monstrosity, whether development is complete or arrested or irregular, are all treated as though they depended on a common cause; and thus much confusion has arisen and misunderstanding.

Many of these deformities are wrongly styled dislocations. Neither was the head of the femur planned to occupy an acetabulum, nor is there an acetabulum to occupy; they are malformations. And

\footnotetext{
2 The Surgical Treatment of the Diseases of Infancy and Childhood. p. 220 .

St. George's Hospital Reports, vol, i, p. 219.

4 Medico-Chirurgical Transactions, vol. xliij, 1860. Holmes' System of Surgery, vol. iv, Hrt. "Intra-uterine Fractures." 1864

Anatomie phthologique du Corps humain, tome

Ueber die Ursacken der Angeborenen Hüftgelenksverrenkungen. Archiv fïr Path., Anat. und Phys., Bd. Ixxiv. p. 303 .
} 